\title{
Changes in regional gray matter volume in women with chronic pelvic pain: A voxel-based morphometry study
}

\section{Citation}

As-Sanie, Sawsan, Richard E. Harris, Vitaly Napadow, Jieun Kim, Gina Neshewat, Anson Kairys, David Williams, Daniel J. Clauw, and Tobias Schmidt-Wilcke. 2012. “Changes in Regional Gray Matter Volume in Women with Chronic Pelvic Pain: A Voxel-Based Morphometry Study." Pain 153 (5) (May): 1006-1014. doi:10.1016/j.pain.2012.01.032.

\section{Published Version}

10.1016/j.pain.2012.01.032

\section{Permanent link}

http://nrs.harvard.edu/urn-3:HUL.InstRepos:36304194

\section{Terms of Use}

This article was downloaded from Harvard University's DASH repository, and is made available under the terms and conditions applicable to Other Posted Material, as set forth at http:// nrs.harvard.edu/urn-3:HUL.InstRepos:dash.current.terms-of-use\#LAA

\section{Share Your Story}

The Harvard community has made this article openly available.

Please share how this access benefits you. Submit a story.

Accessibility 


\title{
Changes in regional gray matter volume in women with chronic pelvic pain - a voxel based morphometry study
}

\author{
Sawsan As-Sanie, MD, MPH ${ }^{1}$, Richard Harris, $\mathrm{PhD}^{2}$, Vitaly Napadow, $\mathrm{PhD}^{3}$, Jieun Kim, \\ $\mathrm{PhD}^{3}$, Gina Neshewat, BS $^{1}$, Anson Kairys, BA $^{2}$, David Williams, $\mathrm{PhD}^{2}$, Daniel Clauw, MD $^{2}$, \\ and Tobias Schmidt-Wilcke, MD ${ }^{2,4}$ \\ ${ }^{1}$ Department of Obstetrics and Gynecology, University of Michigan, Ann Arbor MI 48109 \\ ${ }^{2}$ Department of Anesthesiology, Chronic Pain and Fatigue Research Center, University of \\ Michigan, Ann Arbor, MI 48109
}

${ }^{3}$ Athinoula A. Martinos Center for Biomedical Imaging, Department of Radiology, Massachusetts, General Hospital, Charlestown, MA 02129

${ }^{4}$ Department of Neurology, University of Tübingen, 07071 Tübingen, Germany

\begin{abstract}
Chronic pelvic pain (CPP) is a highly prevalent pain condition, estimated to affect $15-20 \%$ of women in the United States. Endometriosis is often associated with CPP, however other factors, such as pre-existing or concomitant changes of the central pain system, might contribute to the development of chronic pain. We applied voxel-based morphometry to determine whether women with CPP with and without endometriosis display changes in brain morphology in regions known to be involved in pain processing.Four subgroups of women participated: 17 with endometriosis and CPP, 15 with endometriosis without CPP, 6 with CPP without endometriosis, as well as 23 healthy controls. All patients with endometriosis and/or CPP were surgically-confirmed. Relative to controls, women with endometriosis-associated CPP displayed decreased gray matter volume in brain regions involved in pain perception including the left thalamus, left cingulategyrus, right putamen, and right insula. Women with CPP without endometriosis also showed decreases in gray matter volume in the left thalamus. Such decreases were not observed in patients with endometriosis that had no CPP. We conclude thatCPP is associated with changes in regional gray matter volume within the central pain system. Although endometriosis may be an important risk factor for the development of CPP, acting as a cyclic source of peripheral nociceptive input, our data support the notion that changes in the central pain system also play an important role in the development of chronic pain, regardless of the presence of endometriosis.
\end{abstract}

\section{Keywords}

chronic pelvic pain; endometriosis; voxel based morphometry; thalamus; cingulate cortex

(C) 2012 International Association for the Study of Pain. Published by Elsevier B.V. All rights reserved.

Corresponding author: Tobias Schmidt-Wilcke, MD Chronic Pain and Fatigue ResearchCenter Department of Anaesthesiology University of Michigan Ann Arbor, MI, USA tobias.schmidt-wilcke@med.uni-tuebingen.de.

Competing interests D. J. Clauw declares associations with the following companies: Cypress Bioscience, Eli Lilly and Company, Forest Laboratories, Pierre Fabre Médicament, Pfizer, Procter \& Gamble, Novu, Jazz, Johnson and Johnson, Merck, and Wyeth Pharmaceuticals. See the article online for full details of these relationships. The other authors declare no competing interests.

Publisher's Disclaimer: This is a PDF file of an unedited manuscript that has been accepted for publication. As a service to our customers we are providing this early version of the manuscript. The manuscript will undergo copyediting, typesetting, and review of the resulting proof before it is published in its final citable form. Please note that during the production process errors may be discovered which could affect the content, and all legal disclaimers that apply to the journal pertain. 


\section{Introduction}

Chronic pelvic pain (CPP) is defined as "non-cyclic pain of 6 or more months' duration that localizes to the anatomic pelvis, anterior abdominal wall at or below the umbilicus, the lumbosacral back, or the buttocks and is of sufficient severity to cause functional disability or lead to medical care"[3]. CPP is estimated to affect 15-20\% of women in the United States, with direct health-care costs approaching $\$ 2.8$ billion dollars per year[3;23]. It is the primary indication for $10 \%$ of outpatient gynecology visits, $40 \%$ of diagnostic laparoscopies, and $12-17 \%$ of hysterectomies performed annually [19;46].

Despite its high prevalence and negative impact, little is known about the mechanismsunderlying CPP. As in most other chronic pain syndromes, its pathogenesis cannot be entirely explained by the presence or severity of "peripheral pathology." For example, in women with endometriosis-associated CPP, there is little if any association between the severity of pain and the extent of endometriosis[6;27;43].Medical and surgical therapies are not always effective and pain frequently recurs, often without evidence of residual disease[35;38;42]. Against this background, endometriosis must be viewed as an important but insufficient risk factor for the development of CPP.

Pain in many other chronic pain syndromes has been shown to be related to central nervous system (CNS) amplification of pain processing, which often occurs in the absence of injury or inflammation of peripheral structures[8;12;13;15;17;24]. From a neurobiological perspective, the mechanisms contributing to pain amplification and chronicity are heterogeneous and likely occur at various levels of the nervous system. In line with this evidence, structural alterati ons in brain regions associated with pain perception and modulation have also been identified in patients with chronic pain. The most reproducible finding is a decrease in gray matter density/volume in the thalamus, cingulate cortex and the insular cortex (IC) [29;30]. It has been postulated that such changes in regional brain morphology may be responsible not only for the evolution and/or maintenance of the chronic pain state, but might also contribute to other common co-morbid clinical traits, such as mood disorders and cognitive impairment[10;22;32].

Therefore, studies of brain anatomy and function might also be important for understanding the pathogenesis of CPP. The primary aim of this study was to use voxel-based morphometry (VBM) to determine whether women with CPP display changes in regional brain morphologyand whether such changes are present in women with similar pelvic pathology without CPP. We investigated three patient subgroups: CPP and endometriosis, endometriosis but no CPP, and CPP but no endometriosis and compared each to healthy controls. We hypothesized that CPP is associated with decreased gray matter volume in brain regions associated with pain perception and modulation, and that these differences are associated with the experience of chronic pain rather than the presence or absence of endometriosis. If this hypothesis is correct, then CPP patients (with and without endometriosis) should show gray matter changes in structures within the pain system relative to controls, and these changes would not be present in endometriosis patients without CPP.

\section{Methods}

\subsection{Subjects}

Four cohorts of participants were included: 17 women with endometriosis-associated CPP ( $\oplus$ Endo $\oplus$ Pain), 15 women with "pain-free" endometriosis( $\oplus$ Endo $\varnothing$ Pain, for a definition of "pain-free" see below), 6 women with CPP but no evidence of endometriosis $(\varnothing$ Endo $\oplus$ Pain 
group, surgically confirmed), and 26 healthy women (HCs). For details seeTables 1 and 2 and Figure 1. All participants were reproductive age women (18-52 years) who had not undergone prior hysterectomy and/or bilateral oophorectomy. Women with endometriosis were recruited from a tertiary-care endometriosis and pelvic pain referral center, as well as through advertisement to the local community. Inclusion criteria for endometriosis participants included ahistory of surgically confirmed endometriosis within 3 years of study participation. Most participants received their surgical diagnosis at another medical institution. Operative reports were reviewed by a gynecologist with significant experience in the surgical evaluation of endometriosis (SA) blinded to study results, and endometriosis was assigned a stage according to the revised American Fertility Society endometriosis scoring system (rAFS)[2]. Surgical pathology was documented when available but not required for participation since pathologic confirmation was not routinely performed in all participants. Potential participants with a history of endometriosis were screened by a phone interview and were invitedto participate only if they fell within one of two categories of pelvic pain severity: (1) chronic pelvic pain or(2) "pain-free" endometriosis. CPP was defined as moderate to severe pelvic pain that is $\geq 4$ on a $0-10$ verbal rating scale for greater than 6 months duration, and was non-cyclic occurring for at least 14 days of each month, not just limited to the time of menstrual bleeding. Pelvic pain was localized to the anatomic pelvis, and could include but was notlimited to only symptoms of dyspareunia (pain with intercourse), dyschezia (pain with bowel movements), or focal low back pain. "Pain-free" endometriosis was defined as the absence of any prior history of chronic pelvic pain and the absence of significant dysmenorrhea, defined as pelvic pain during menstruation that is $\geq 4$ on a 0-10 verbal rating scale occurring for 5 or more days of each menstrual cycle(i.e. all study participants falling into the $\oplus$ Endo $\varnothing$ Pain group had at maximum 4 days of mild pain associated with menses). This study also included data on six participants with CPP but no evidence of endometriosis ( $\varnothing$ Endo $\oplus$ Pain). These women fulfilled all clinical criteria of $\mathrm{CPP}$, had no prior surgical history of endometriosis, had undergone a diagnostic laparoscopy within 3 years of study participation and had no surgical evidence of endometriosis or pelvic adhesions at the time of surgical exploration. No other anatomic sources of pain could be identified in these patients.

Using standardized criteria, all women with endometriosis and/or CPP were formally screened for and excluded from participation if they had one or more of the following chronic pain syndromes thought to be associated with a central nervous system abnormality in pain processing: fibromyalgia, chronic fatigue syndrome, interstitial cystitis, chronic low back pain unrelated to pelvic pain, or temporomandibular disorder.Women with CPP had to discontinue opioid analgesia for 72 hours prior to the MRI visit.

HCs completed standardized case report forms to assess their medical history, surgical history, medication use, and any pain symptoms. All HCs were pain-free women withoutsymptoms of dysmenorrhea, no known history of endometriosis, no history of chronic pain (including pelvic pain or discomfort).All HCs were formally screened in a similar fashion to endometriosis participants, and did not meet criteria for any of the chronic pain syndromes previously defined, and did not have a history of chronic, recurrent headaches or irritable bowel syndrome. Women with current symptoms of major depressive disorder, bipolar disorder, general anxiety disorder (according to Diagnostic and Statistical Manual of the American Psychiatric Association IV criteria)[1] and those currently on antidepressants for any indication were excluded.HCs were recruited from ongoing studies using the same functional MRI protocol used in this study. Because the mean age of endometriosis/CPP subgroups wassignificantlydifferent,each subgroup of endometriosis/ CPP patients was compared to an age-matched subset of HCs in a 1:1 or 2:1 ratio (Figure 1). 
All participants had to be free of contraindications for an MRI study as determined by a health questionnaire and were right-handed to simplify brain mapping. In order to minimize the influence of menstrual cycle variability on study results, all study visits were performed between days 2-10 of the menstrual cycle in women who were not using hormonal contraceptives. Additional exclusion criteria for all participants includeda severe physical impairment (e.g., complete blindness, deafness, paraplegia), co-existing physical injury (e.g. sprained ankle, neck injury etc.), co-morbid medical illnesses (e.g. morbid obesity, autoimmune diseases, cardiopulmonary disorders, uncontrolled endocrine or allergic disorders or malignancy within 2 years), any present psychiatric disorder involving a history of psychosis, current suicide risk or attempt within 2 years of the study, substance abuse within 2 years, a pending status associated with disability or the receipt of disability compensation, being pregnant, lactating, or menopausal (defined as no menses for greater than one year unrelated to exogenous hormonal suppression), or a contraindication to undergoing MRI (e.g. metal implants, claustrophobia).

\subsection{Clinical and experimental pain}

Participants with endometriosis and/or CPP completed additional standardized case report forms to assess their surgical history, medication use, and the severity, pattern and characteristics of their pelvic pain. Measurements included numeric ratings (0-10) of pelvic pain during their menses, and the average pelvic pain intensity and unpleasantness in the last month measured with the Gracely Box scale(Figure 2)[14].The GBS is a numerical scale that is used to evaluate pain intensity and unpleasantness (GBS pelvic pain intensity and unpleasantness). This scale is comprised of 21 boxes, sequentially numbered beginning with 0 and ending with 20. It is aligned vertically, with 0 representing the least amount of pain. Descriptive words are arranged next to the numbers corresponding with increasing levels of pain intensity and unpleasantness.

Prior to MRI scanning,experimental pain testing was conductedon all participants using the multiple random staircase (MRS) method previously described by our group[15]. Pressurepain values required to elicit faint pain ( 0.5 on the GBS, see below and Figure2), mild pain (7.5 on the GBS), and slightly intense pain (13.5 on GBS) pain were determined for every subject by applying associatedpressures $\left(1-10 \mathrm{~kg} / \mathrm{cm}^{2}\right.$ to the right or left thumb)as determined by the MRS method.

\subsection{Depression, anxiety, and physical function}

All participants completed standardized measures of depression, anxiety, and physical function. These values were used tocharacterizethe degree of psychological distress and function of patient subgroups relative to healthy controls, and to determine if such measures of distress correlate with changes in regional GM volume. Depressive symptoms were measured with the Center for Epidemiological Studies-Depression Scale (CES-D), a 20-item self-report inventory designed to assess depressive mood[28]. Participants are asked to indicate how frequently they experience each set of symptoms during the past week. The total possible score, ranging from 0-60, reflects both the number of symptoms and the frequency of their occurrence. Trait anxiety was measured using the 10-item Trait Anxiety scale fromthe State-Trait Personality Inventory (STPI)[36]. Scores range from 10 to 40, with higher values indicating higher anxiety symptoms. Physical function and health status were measured with the SF-36, and provides individual summary scores for physical function and mental function[45]. The summary scores have been standardized to have a mean $=50$, $\mathrm{SD}=10$ in the general US population, with larger values indicating better function. All questionnaires were administered within 72 hours of the MRI scan. 
Group differences between characteristics of patients and controls and subgroups of patients were evaluated using Student's t-test, and Pearson's Chi-square test, as appropriate. The significance threshold was set at $\mathrm{p}<0.05$. Overall group differences between characteristics of three subgroups of endometriosis and pelvic pain patients were evaluated with analysis of variance and Pearson's Chi-square tests, as appropriate. Further analysis between subgroups of patients $(\oplus$ Endo $\varnothing$ Pain vs. $\oplus$ Endo $\oplus$ Pain; $\oplus$ Endo $\varnothing$ Pain vs. $\varnothing$ Endo $\oplus$ Pain; and $\bigoplus$ Endo $\oplus$ Pain vs. $\varnothing$ Endo $\oplus$ Pain) was performed with Student's t-test and Pearson's Chisquare tests. Results were thresholded at $\mathrm{p}<0.05$, after performing a domain specific correction for multiple comparisons (Bonferroni; $\mathrm{p}<0.05 /$ no. of domains), yielding a threshold $\mathrm{p}<0.017$ for three domains. Three domains included clinical pain, experimental pain testing, and measures of mood/function.

\subsection{Scanning protocol}

Magnetic resonance imaging was performed on a 3.0 Tesla GE Signa scanner (LX [VH3] release, Neuro-optimized gradients). For each subject, a T-1 weighted gradient echo data set (TR $1400 \mathrm{~ms}$, TE $5.5 \mathrm{~ms}$, flip angle $20^{\circ}$, FOV $256 \times 256$, yielding 124 sagittal slices with a defined voxel size of $1 \times 1 \times 1.2 \mathrm{~mm}$ ) was acquired. An Eclipse 3.0 T 94 quadrature head coil was used. Inspection of individual T1 MR-images revealed no gross morphological abnormality for any participant.

\subsection{Pre-processing and statistical analysis of VBM data}

The SPM5 software package (Functional Imaging Laboratories, London, UK) running under Matlab $7 \mathrm{~b}$ was used to pre-process and analyze structural data.[5]Estimation of total gray matter volume (GMV), total white matter volume (WMV) and cerebrospinal fluid (CSF) was performed by segmenting the original image into GM, WM and CSF, using the Ibaspm toolbox (toolbox for automatic parcellation of brain structures), provided by the Cuban Neuroscience Center[4].

Pre-processing of structural images for VBM analyses was performed using the VBM toolbox (VBM 5.1, provided by C. Gaser, default settings), which involved spatial normalization, segmentation and spatial smoothing (Gaussian kernel of $8 \mathrm{~mm}$ full-width at half maximum for GM images). Modulated images were used for statistical analyses; correspondingly, GM and WM values are referred to as regional GM or WM volume.Significant regional differences in gray matter values between groups were identified applying voxel-wise statistics within the general linear model (two sample t-test with age as nuisance variable, also referred to as parametric cohort analysis).

As the $\oplus$ Endo $\oplus$ Pain and the $\oplus$ Endo $\varnothing$ Pain group were 10 years apart in age (group average), each group was analyzed with its own, age adjusted healthy control (HC) group, i.e for the $\oplus$ Endo $\oplus$ Pain group $(n=17)$ and for the $\oplus$ Endo $\varnothing$ Pain $(n=15)$. Each of these two $\mathrm{HC}$ groups were drawn from the pool of $23 \mathrm{HCs} .8 \mathrm{HC}$ participantswere included in both control groups. For the six $\varnothing$ Endo $\oplus$ Painpatients, wematched $12 \mathrm{HCs}$ of the same age, 8 of which were included in the previous HC comparison groups. With respect to the small number of patients in this group,a non-parametric group comparison was performed using the SnPM (Statistical nonParametric Mapping) toolbox, which uses the general linear model and voxel-wise statistics to construct pseudo t-statistic images, and then assesses the data for significance using a non-parametric multiple comparison procedure based on permutation testing (in our case 5000 permutations). Permutation tests are recommended for designs with low degrees of freedom[25].The following design module was applied: 2 groups; twosample t-test; 1 scan per subject,controlling for age.To avoid possible edge effects around the border between gray and white matter and to include only relatively homogeneous 
voxels, we excludedall voxels with a GM matter value of $<0.1$ (maximum value of 1 ) for both parametric and non-parametric tests.

Statistical maps were corrected for multiple comparisons on the cluster level $(\mathrm{p}<0.05$, derived from an uncorrected voxel level threshold of $\mathrm{p}<0.001$, with a cluster extent of 660 contiguous voxels), as estimated byAlphaSim, an application implemented in the Analysis of Functional NeuroImages (AFNI) software (http://afni.nimh.nih.gov/afni/doc/manual/ AlphaSim), which is based on a Monte Carlo simulation (5000 simulations) applied to a whole brain mask (including cortical gray matter, white matter, CSF, brainstem and cerebellum). As we had a clearly defined a priori hypothesis, looking for GM changes within structures of the pain system and pain modulatory system (midbrain, thalamus, putamen, amygdala, somatosensory cortex, insular cortex, cingulate cortex and prefrontal cortex), we allowed in a second step for these regions a relaxed cluster extent threshold of 200 contiguous voxels. Clusters with an extent between 200 and 660 voxels outside the pain system are reported as these results could be interesting for future analyses, but will not be further commented on (and should be regarded as uncorrected). Anatomical labeling of brain regions was performed using the SPM5 extension xjview(http://www.alivelearn.net/ xjview8/).

To further explorebehavioral relevance of changes in regional GM volume, we performed correlation analyses, extracting the eigenvariate from the GM clusters identified in the cohort analyses. This yielded an average GM value for that region in each person; values were then transferred to SPSS (Version 17), where we performed correlation analyses, correlating extracted GM values with 3 domains of behavioral and pain data:

1. clinical pain: pain intensity (VAS) on day of fMRI scan,GBS pelvic pain intensity and GBS pelvic pain unpleasantness in the last month, and duration of CPP.

2. experimental pain: pressure needed to elicit faint ( 0.5 on the GBS), mild (7.5 on the GBS) and/or slightly intense pain (13.5 on the GBS),

3. mood and physical function measures: anxiety, depression and physical function component of SF-36.

For the $\varnothing$ Endo $\oplus$ Pain group ( $n=6)$ we performed Spearman's rank correlations due to the small sample size; within the other two groups ( $\oplus$ Endo $\oplus$ Pain and $\varnothing$ Endo $\oplus$ Pain), Pearson correlations were performed. Results were thresholded at $\mathrm{p}<0.05$, after performing a domain specific correction for multiple comparisons (Bonferroni; $p<0.05 /$ no.ofdomains), yielding a threshold $\mathrm{p}<0.017$ for all three domains. For explorative reasons, correlation analyses were also performed within each group between GM eigenvariates extracted from GM clusters, identified in the cohort analyses, since correlations between GM values in remote brain regions can be an indicator of structural connectivity[18].No corrections for multiple comparisons were applied in these analyses.

\section{Results}

\subsection{Behavioral data: age, pain, pain thresholds, anxiety and depression}

Descriptive data on age, race, and current hormonal contraceptive use is presented in Table 1. Generally, participants with CPP, both with and without endometriosis $(\oplus$ Endo $\oplus$ Pain and $\varnothing$ Endo $\oplus$ Pain), were young women who were significantly younger than women with endometriosis without CPP.

The surgical history and clinical pain experience of women with endometriosis and/or CPPare presented in Table 2. Although more advanced stage endometriosis was found in the $\bigoplus$ Endo $\varnothing$ Pain group when compared to the $\oplus$ Endo $\oplus$ Pain group, there was no correlation 
between endometriosis stage and any other clinical pain characteristic (data not presented). When comparing the two subgroups of participants with $\mathrm{CPP}(\oplus$ Endo $\oplus$ Pain and $\varnothing$ Endo $\oplus$ Pain), there was also no difference in median duration of pelvic pain symptoms $(\mathrm{p}=0.29)$, average number of pain days per month $(\mathrm{p}=0.42)$, and mean pain intensity before $(\mathrm{p}=0.91)$ or during menses $(\mathrm{p}=0.98)$.

\subsection{Results from voxel based morphometry - cohort analyses and correlations analyses}

VBM analyses revealed several regions of GM volume changes in various parts of the brain, while comparing each group to their own matched $\mathrm{HC}$ group. In the $\oplus$ Endo $\oplus$ Pain group, GM volume decrease (in comparison to the $\mathrm{HC}$ group) was observed in the left thalamus, left middle frontal gyrus (MFG), bilateral mid cingulate cortex (MCC), right putamen, and right insular cortex. An increase in regional GM volume was observed in the left amygdala. For details seeTable 3 and Figure 3.

Correlation analyses within the $\oplus$ Endo $\oplus$ Pain were based on 16 patients, since clinical pain data from one patient were missing. GM volume decrease in the cingulate gyrus (GM values extracted from the cluster in the MCC identified in the group comparison) correlated with regional GM volume of theleft thalamus $(r=0.56, p=0.024)$ andwith the regional GM volume of the left MFG $(r=0.60, p=0.014)$. GM valuesextracted from the MCC cluster correlated negatively with pain unpleasantness $(r=-0.59, p=0.016)$, i.e.the less GM volume, the more unpleasant pain was perceived); surprisingly it alsocorrelated negatively with pressure needed to elicit high pain $(r=-0.60, p=0.013)$, i.e. the less GM volume, the more pressure was needed to elicit a pain score of 13.5 on the GBS. GM values extracted from the thalamus cluster and from the left MFG cluster correlated with MCC GM values (see above) and with pain unpleasantness (rthalamus $=-0.64, \mathrm{p}=0.008 ; 1_{\mathrm{MFG}}=-0.53, \mathrm{p}=$ 0.037 , also see Figure 4). Finally GM values extracted from the cluster found in the right posterior IC also correlated negatively with pain unpleasantness $\left(\mathrm{r}_{\mathrm{IC}}=-0.59, \mathrm{p}=\right.$ $0.017)$.Neither anxiety nor depression scores were significantly correlated with GM values of any of the clusters. There was also no significant correlation between pain duration and GM volume in any cluster.

In the $\oplus$ Endo $\varnothing$ Pain group decreased GM volume (relative to HCs) was only observed in the right inferior temporal gyrus. Furthermore, this group showed an increase in regional GM volume in several regions including the right PAG, right inferior frontal gyrus (IFG), and right MFG. For details see Table 3 and Figure 3. There was a trend for a positive correlation between pressure needed to elicit mild pain (7.5 on the GBS)andregional GM volume in the right PAG cluster (identified in the group comparison with $\mathrm{HCs}(\mathrm{r}=0.53, \mathrm{p}=0.042)$, i.e the higher the GM valuesthe more pressure was needed to elicit mild pain.Regional GM values inthe right MFG correlated positively with regional PAG GM values $(r=0.54, p=0.04)$ and also with pressure needed to elicit low pain $(0.5$ on the GBS, $r=0.60, p=0.019)$.

In the $\varnothing$ Endo $\oplus$ Pain group, GM volume decrease was observed in the left thalamus when compared to HCs. There was a highly significant negative correlation between regional left thalamus volume and pain ratings during menstruation $(r=-0.94, p=0.003)$. Again, there was no significant correlation between pain duration and GM volume in any cluster in the $\varnothing$ Endo $\oplus$ Pain group.

\section{Discussion}

The current study sought to investigate changes in regional brain morphology in patients with CPP, with and without endometriosis, as an attempt to disentangle the interaction between chronic pain, endometriosis and changes in brain morphology. A decrease in regional GM volume in the thalamus was found in patients with CPP, regardless of the 
presence of endometriosis. Furthermore, patients with CPP and endometriosis showed decreased GM volume in the right posterior insula, the right putamen and the MCC. Decreases in GM in the thalamus, MCC and IC correlated with pain unpleasantness in this group. Endometriosis patients without CPPshowed no evidence of a GM decrease within the pain system, instead an increase in regional GM volume was observed in the mesencephalon (PAG) and the right prefrontal cortex.

Our results are well in line with other VBM studies reporting decreases in GM in chronic pain patients, including other pelvic pain conditions, in regions of the pain system (thalamus, IC, CC) and/or those involved in pain modulation (prefrontal cortex) [7;9;11;33;40]. Interestingly, a similar pattern of regional GM decrease has been observed in patients suffering from IBS, with decreased volume in various cortical and subcortical structures, such as the ACC, MCC, IC, prefrontal cortex, putamen andthalamus [7;9;34]. Seminovicz et al. described decreases in GM volume in the thalamus bilaterally in IBS patients, close to where thalamic GM decrease was found in this study. Although our images do not have sufficient resolution to distinguish nuclei, the thalamic GM decrease seems to involve the medial nuclei. These nuclei are structurally connected with the PFC and the anterior IC and have been proposed to be part of a visceral pain network. Interestingly in the $\oplus$ Endo $\oplus$ Pain group, regional GM volume in the thalamus correlated with regional MCC volume (and both were negatively correlated with pain unpleasantness), suggesting that pain in this cohort involves a network[18]. It is conceivable that both pain syndromes, CPP and IBS, share a common underlying pathophysiology resulting in disturbed visceroception, i.e. (re)organisation of the thalamus allows normally "silent" visceral/proprioceptive signals to be processed differently, gain (increased) access to higher cortical structures, and be perceived as painful.

Particularly relevant to this study is the growing line of evidence that altered brain morphology and function is already present in patients with dysmenorrhea[40;41;44], which is often reported prior to transitioning to CPP. Against this background, dysmenorrhea can be considered a precursor stage for some women who progress to CPP. For example, Vincent et al. reported altered CNS response to noxious stimuli in women with dysmenorrhea that persists beyond the time of menstruation, when women do not report pain [44].Tu et al. described changes in regional cerebral metabolism in women with dysmenorrheain the thalamus bilaterally[41].These women also displayed increases in GM volume in the MCC, secondary somatosensory cortex, hippocampus, hypothalamus and mesencephalon [40]. Interestingly the increase in the MCC projected near the cluster of GM decrease found in our study, whichhas been described in other chronic pain states[21;30]. Furthermore, the regional increase in GM in the mesencephalon matched the increase in GM found in the $\oplus$ Endo $\varnothing$ Pain group in the current study; both clusters projected to the PAG, a key structure in the antinociceptive system. As such, it is tempting to hypothesize that despite endometriosis serving as a cyclic pain generator, the $\oplus$ Endo $\varnothing$ Pain group experienced little if any (cyclic) pain, due to adaptive changes in the PAG, possibly due to increased antinociceptive capacity.In support of this hypothesis, we found that PAG volume showed a trend to be positively correlated with pressure needed to elicit mild pain, suggesting a relationship between PAG volume and pressure pain thresholds.

\section{Endometriosis, dysmenorrhea and CPP - a conceptual approach to pain chronification}

Endometriosis is an important risk factor for CPP and it is well accepted that endometriosis can act as a nociceptive source and peripheral pain generator during menses often leading to cyclic pelvic pain (dysmenorrhea). However it remains unclear why only some women undergo a transition to a chronic pain state, while others do not. One might argue that endometriosis is the only source of pain, and that the pain experienced in these women outside of the menses is due to an ongoing neuroinflammatory process in the pelvis. In such 
a scenario, chronic pain is conceptually understood as being the result of an ongoing nociceptive input (defined here asa peripherally generated, neural input usingnociceptive pathways, such as $\mathrm{C}$ and $\mathrm{A} \delta$ fiber) to an otherwise normal brain. However, this hypothesis has recently beenchallengedby several authors who summarize the increasing evidence that the mechanism of pelvic pain in women with endometriosis may be partly related to CNS amplification of pain processing[20;37]. Thisprocess of central pain modulation (amplification or inhibition) could explain why some women suffer from dysmenorrhea and/ or CPP but do not have an identifiable peripheral nociceptive input, while other women with endometriosis (even severe) experience little if any pain.

The hallmark of chronic pain seems to be a decrease in GM volume in structures known to be part of the pain system; although increases in regional GM have been described in some studies, mainly in the basal ganglia[31;33;47]. These changes in GMvolume may vary between pain states and brain structures involved, and each might depend on pain duration, pain occurrence (intermittent vs persistent), personality traits and medication; as such the current literature is inconclusive. However, it is also possible that GM changes are dynamic and change over time within an individual. For example, some recent longitudinal studies suggest that GM changes can be induced by repeated experimental pain [39], and that changes associated with clinical pain can reverse spontaneously [26] and/or after removal of the nociceptive source [16]. Given thatchanges in regional brain morphology arenot preexisting in the few published longitudinal studies, but develop afterrepeated nociceptive input, it is possible that the initial 'reaction' of the brain is a GM increase in certain pain transmitting areas, such as the MCC and the somatosensory cortex, as well as changes in regional metabolism (thalamus)[39-41]. This initial increase in GM might at first be an adaptive mechanism. Dependent on the duration and persistence of the nociceptive input, local characteristics of the neural tissue and other factors such as capacity/effectiveness of antinociceptive systems, a transition to a chronic pain state might then take place in some patients, which is marked by a decrease in regional gray matter volume in the pain system. These changes, once they occur, may then contribute to an ongoing pain perception, even after disappearance/removal of the initial nociceptive source.

Whether pain becomes chronic depends on the interaction of various factors, namely the persistence of the peripheral pain generator, the antinociceptive capacity and (maladaptive) neuroplasticity of the pain system. Given that dysmenorrhea is often a pre-stage of CPP, our data and those of others suggest that pain chronification is marked by a decrease in regional GM volume in the pain system. Those women that remain relatively "pain-free" on the other hand do not show these decreases. In contrast, they show an increase in GM volume in the antinociceptive system, which again might be adaptive. A conceptual model summarizing the possible relationship between dysmenorrheaand factors associated with the progression to $\mathrm{CPP}$ is presented in Figure 5.

\section{Limitations}

The group of $\varnothing$ Endo $\oplus$ Pain group was rather small $(n=6)$. Although we tried to address this issue by performing non-parametric tests, a larger sample size is preferable. The small sample in this subgroup is due to the fact that endometriosis is highly prevalentin the CPPclinic that referred patients to this research protocol $(70-80 \%)$ and the strict inclusion criteria that were required of this subgroup. For obvious reasons, not all of the HCs had been explored via laparoscopy. Given a general population prevalence of endometriosis of 5-10\%, we estimate that 1-2 HCs had undiagnosed endometriosis. However, given this small number and substantial differences seen between patient and HC subgroups, we would not expect this small misclassification error to significantly influence our results.Finally, the cross-sectional design of this study precludes the ability to determine whether the changes in 
GM volume are a cause or consequence of the pain experience. Longitudinal studies are needed to better address this important question.

\section{Conclusions}

The current study adds to the growing body of literature suggesting that chronic pain states are associated with changes in regional brain morphology. Our data challenge the idea of endometriosis being the only and direct cause of pelvic pain in women with endometriosisassociated CPP, suggesting that central mechanisms as reflected by changes in regional brain morphology, such as thalamic GM decrease, plays a pivotal role. Whether in the wake of a prolonged nociceptive input (caused by endometriosis) or preexisting, these central mechanisms seem to play a critical role in the pain chronification process. As such, we suggest that CPP in most women results from an interaction between antinociceptive capacity and central (mal)adaptive plasticity.

\section{Acknowledgments}

This work was supported in part by the following research grants: NIH Building Interdisciplinary Research in Women's Health K12HD001438, NIH UL1RR024986, Bayer Droegemueller Award in Clinical Research, NIH R01-AR050044, and DAMD 17-00-2-0018.Tobias Schmidt-Wilcke is currently supported by a grant of the DFG (Deutsche Forschungsgemeinschaft, GZ: SchM 2665/1-1).Richard Harris is supported by grants from the Dana foundation and the Department of Defense (Army grant: DAMD-W81XWH-07-2-0050). VitalyNapadow and Jieun Kim were supported by NCCAM, NIH (R01-AT004714 (Napadow), P01-AT002048 (Rosen)).

\section{Literature}

[1]. American Psychiatric Association. Diagnostic and statistical manual of mental disorders. Revised 4th ed. Author; Washington, DC: 2000.

[2]. Revised American Society for Reproductive Medicine classification of endometriosis: 1996. Fertil Steril. 1997; 67(5):817-821. [PubMed: 9130884]

[3]. ACOG Practice Bulletin No. 51. Chronic pelvic pain. Obstet Gynecol. 2004; 103(3):589-605. [PubMed: 14990428]

[4]. Alemán-Gómez, Y.; Melie-García, L.; V-H, P. IBASPM: Toolbox for automatic parcellation of brain structures. Presented at the 12th Annual Meeting of the Organization for Human Brain Mapping; Florence, Italy. June 11-15, 2006; Available on CD-Rom in NeuroImage

[5]. Ashburner J, Friston KJ. Unified segmentation. Neuroimage. 2005; 26(3):839-851. [PubMed: 15955494]

[6]. Balasch J, Creus M, Fabregues F, Carmona F, Ordi J, Martinez-Roman S, Vanrell JA. Visible and non-visible endometriosis at laparoscopy in fertile and infertile women and in patients with chronic pelvic pain: a prospective study. Hum Reprod. 1996; 11(2):387-391. [PubMed: 8671229]

[7]. Blankstein U, Chen J, Diamant NE, Davis KD. Altered brain structure in irritable bowel syndrome: potential contributions of pre-existing and disease-driven factors. Gastroenterology. 2010; 138(5):1783-1789. [PubMed: 20045701]

[8]. Clauw DJ, Schmidt M, Radulovic D, Singer A, Katz P, Bresette J. The relationship between fibromyalgia and interstitial cystitis. J Psychiatr Res. 1997; 31(1):125-131. [PubMed: 9201654]

[9]. Davis KD, Pope G, Chen J, Kwan CL, Crawley AP, Diamant NE. Cortical thinning in IBS: implications for homeostatic, attention, and pain processing. Neurology. 2008; 70(2):153-154. [PubMed: 17959767]

[10]. Du MY, Wu QZ, Yue Q, Li J, Liao Y, Kuang WH, Huang XQ, Chan RC, Mechelli A, Gong QY. Voxelwise meta-analysis of gray matter reduction in major depressive disorder. Prog Neuropsychopharmacol Biol Psychiatry. 2011

[11]. Farmer MA, Chanda ML, Parks EL, Baliki MN, Apkarian AV, Schaeffer AJ. Brain Functional and Anatomical Changes in Chronic Prostatitis/Chronic Pelvic Pain Syndrome. J Urol. 2011 
[12]. Giesecke J, Reed BD, Haefner HK, Giesecke T, Clauw DJ, Gracely RH. Quantitative sensory testing in vulvodynia patients and increased peripheral pressure pain sensitivity. Obstet Gynecol. 2004; 104(1):126-133. [PubMed: 15229011]

[13]. Giesecke T, Gracely RH, Grant MA, Nachemson A, Petzke F, Williams DA, Clauw DJ. Evidence of augmented central pain processing in idiopathic chronic low back pain. Arthritis Rheum. 2004; 50(2):613-623. [PubMed: 14872506]

[14]. Gracely RH, Kwilosz DM. The Descriptor Differential Scale: applying psychophysical principles to clinical pain assessment. Pain. 1988; 35(3):279-288. [PubMed: 3226757]

[15]. Gracely RH, Petzke F, Wolf JM, Clauw DJ. Functional magnetic resonance imaging evidence of augmented pain processing in fibromyalgia. Arthritis Rheum. 2002; 46(5):1333-1343. [PubMed: 12115241]

[16]. Gwilym SE, Filippini N, Douaud G, Carr AJ, Tracey I. Thalamic atrophy associated with painful osteoarthritis of the hip is reversible after arthroplasty: a longitudinal voxel-based morphometric study. Arthritis Rheum. 2010; 62(10):2930-2940. [PubMed: 20518076]

[17]. Gwilym SE, Keltner JR, Warnaby CE, Carr AJ, Chizh B, Chessell I, Tracey I. Psychophysical and functional imaging evidence supporting the presence of central sensitization in a cohort of osteoarthritis patients. Arthritis Rheum. 2009; 61(9):1226-1234. [PubMed: 19714588]

[18]. He Y, Dagher A, Chen Z, Charil A, Zijdenbos A, Worsley K, Evans A. Impaired small-world efficiency in structural cortical networks in multiple sclerosis associated with white matter lesion load. Brain. 2009; 132(Pt 12):3366-3379. [PubMed: 19439423]

[19]. Howard FM. The role of laparoscopy in chronic pelvic pain: promise and pitfalls. Obstet Gynecol Surv. 1993; 48(6):357-387. [PubMed: 8327235]

[20]. Howard FM. Endometriosis and mechanisms of pelvic pain. J Minim Invasive Gynecol. 2009; 16(5):540-550. [PubMed: 19835795]

[21]. Kuchinad A, Schweinhardt P, Seminowicz DA, Wood PB, Chizh BA, Bushnell MC. Accelerated brain gray matter loss in fibromyalgia patients: premature aging of the brain? J Neurosci. 2007; 27(15):4004-4007. [PubMed: 17428976]

[22]. Luerding R, Weigand T, Bogdahn U, Schmidt-Wilcke T. Working memory performance is correlated with local brain morphology in the medial frontal and anterior cingulate cortex in fibromyalgia patients: structural correlates of pain-cognition interaction. Brain. 2008; 131(Pt 12): 3222-3231. [PubMed: 18819988]

[23]. Mathias SD, Kuppermann M, Liberman RF, Lipschutz RC, Steege JF. Chronic pelvic pain: prevalence, health-related quality of life, and economic correlates. Obstet Gynecol. 1996; 87(3): 321-327. [PubMed: 8598948]

[24]. Naliboff BD, Derbyshire SW, Munakata J, Berman S, Mandelkern M, Chang L, Mayer EA. Cerebral activation in patients with irritable bowel syndrome and control subjects during rectosigmoid stimulation. Psychosom Med. 2001; 63(3):365-375. [PubMed: 11382264]

[25]. Nichols TE, Holmes AP. Nonparametric permutation tests for functional neuroimaging: a primer with examples. Hum Brain Mapp. 2002; 15(1):1-25. [PubMed: 11747097]

[26]. Obermann M, Nebel K, Schumann C, Holle D, Gizewski ER, Maschke M, Goadsby PJ, Diener HC, Katsarava Z. Gray matter changes related to chronic posttraumatic headache. Neurology. 2009; 73(12):978-983. [PubMed: 19770474]

[27]. Porpora MG, Koninckx PR, Piazze J, Natili M, Colagrande S, Cosmi EV. Correlation between endometriosis and pelvic pain. J Am Assoc Gynecol Laparosc. 1999; 6(4):429-434. [PubMed: 10548700]

[28]. Radloff LS. The CES-D Scale: A self-report depression scale for research in the general population. Applied Psychological Measurement. 1977; 1:385-401.

[29]. Schmidt-Wilcke T, Ganssbauer S, Neuner T, Bogdahn U, May A. Subtle grey matter changes between migraine patients and healthy controls. Cephalalgia. 2008; 28(1):1-4. [PubMed: 17986275]

[30]. Schmidt-Wilcke T, Leinisch E, Straube A, Kampfe N, Draganski B, Diener HC, Bogdahn U, May A. Gray matter decrease in patients with chronic tension type headache. Neurology. 2005; 65(9):1483-1486. [PubMed: 16275843] 
[31]. Schmidt-Wilcke T, Luerding R, Weigand T, Jurgens T, Schuierer G, Leinisch E, Bogdahn U. Striatal grey matter increase in patients suffering from fibromyalgia-- a voxel-based morphometry study. Pain. 2007; 132(Suppl 1):S109-116. [PubMed: 17587497]

[32]. Schmidt-Wilcke T, Wood P, Lurding R. Cognitive impairment in patients suffering from fibromyalgia. An underestimated problem. Schmerz. 2010; 24(1):46-53. [PubMed: 20108102]

[33]. Schweinhardt P, Kuchinad A, Pukall CF, Bushnell MC. Increased gray matter density in young women with chronic vulvar pain. Pain. 2008; 140(3):411-419. [PubMed: 18930351]

[34]. Seminowicz DA, Labus JS, Bueller JA, Tillisch K, Naliboff BD, Bushnell MC, Mayer EA. Regional gray matter density changes in brains of patients with irritable bowel syndrome. Gastroenterology. 2010; 139(1):48-57. e42. [PubMed: 20347816]

[35]. Shakiba K, Bena JF, McGill KM, Minger J, Falcone T. Surgical treatment of endometriosis: a 7year follow-up on the requirement for further surgery. Obstet Gynecol. 2008; 111(6):1285-1292. [PubMed: 18515510]

[36]. Spielberger, CD.; Gorsuch, RL. Manual for the State-Trait Anxiety Inventory: (STAI) "(SelfEvaluation Questionnaire)". Consulting Psychologists; Palo Alto: 1979. Vol. Press

[37]. Stratton P, Berkley KJ. Chronic pelvic pain and endometriosis: translational evidence of the relationship and implications. Hum Reprod Update. 2011; 17(3):327-346. [PubMed: 21106492]

[38]. Sutton CJ, Ewen SP, Whitelaw N, Haines P. Prospective, randomized, double-blind, controlled trial of laser laparoscopy in the treatment of pelvic pain associated with minimal, mild, and moderate endometriosis. Fertil Steril. 1994; 62(4):696-700. [PubMed: 7926075]

[39]. Teutsch S, Herken W, Bingel U, Schoell E, May A. Changes in brain gray matter due to repetitive painful stimulation. Neuroimage. 2008; 42(2):845-849. [PubMed: 18582579]

[40]. Tu CH, Niddam DM, Chao HT, Chen LF, Chen YS, Wu YT, Yeh TC, Lirng JF, Hsieh JC. Brain morphological changes associated with cyclic menstrual pain. Pain. 2010; 150(3):462-468. [PubMed: 20705214]

[41]. Tu CH, Niddam DM, Chao HT, Liu RS, Hwang RJ, Yeh TC, Hsieh JC. Abnormal cerebral metabolism during menstrual pain in primary dysmenorrhea. Neuroimage. 2009; 47(1):28-35. [PubMed: 19362153]

[42]. Vercellini P, Somigliana E, Vigano P, De Matteis S, Barbara G, Fedele L. The effect of secondline surgery on reproductive performance of women with recurrent endometriosis: a systematic review. Acta Obstet Gynecol Scand. 2009; 88(10):1074-1082. [PubMed: 19707899]

[43]. Vercellini P, Trespidi L, De Giorgi O, Cortesi I, Parazzini F, Crosignani PG. Endometriosis and pelvic pain: relation to disease stage and localization. Fertil Steril. 1996; 65(2):299-304. [PubMed: 8566252]

[44]. Vincent K, Warnaby C, Stagg CJ, Moore J, Kennedy S, Tracey I. Dysmenorrhoea is associated with central changes in otherwise healthy women. Pain. 2011; 152(9):1966-1975. [PubMed: 21524851]

[45]. Ware, J.; Kosinski, M.; Keller, MD. SF-36 physical and mental health summary scales: a user's manual. The Health Institute; Boston: 1994.

[46]. Whiteman MK, Hillis SD, Jamieson DJ, Morrow B, Podgornik MN, Brett KM, Marchbanks PA. Inpatient hysterectomy surveillance in the United States, 2000-2004. Am J Obstet Gynecol. 2008; 198(1):34, e31-37. [PubMed: 17981254]

[47]. Younger JW, Shen YF, Goddard G, Mackey SC. Chronic myofascial temporomandibular pain is associated with neural abnormalities in the trigeminal and limbic systems. Pain. 2010; 149(2): 222-228. [PubMed: 20236763] 


\section{Chronic Pelvic Pain}

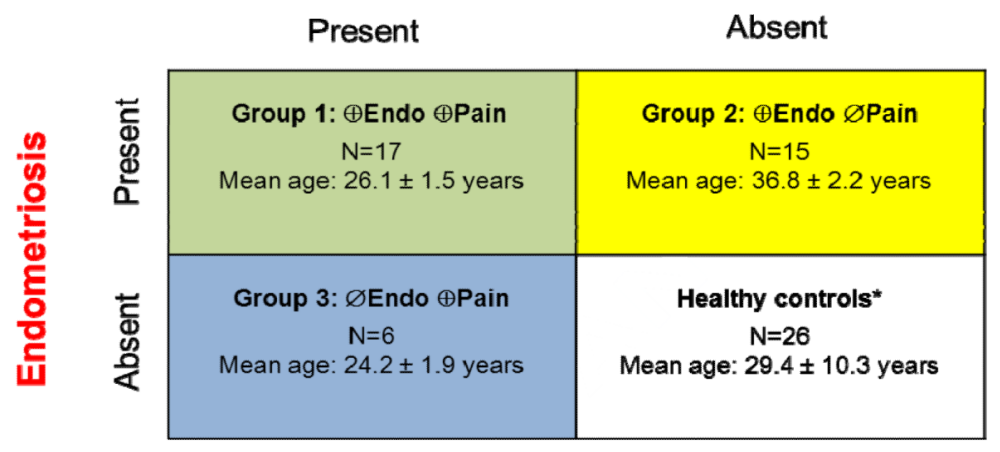

Figure 1. Overview of study population

$\mathrm{N}=$ number; $\oplus$ Endo $\oplus$ Pain = endometriosis with chronic pelvic pain; $\oplus$ Endo $\varnothing$ Pain $=$ endometriosis without chronic pelvic pain, $\varnothing$ Endo $\oplus$ Pain = chronic pelvic pain without endometriosis. * Each subgroup of endometriosis/chronic pelvic pain group (groups 1-3) was compared to an age-matched subset of healthy controls. 


\begin{tabular}{|c|c|c|c|}
\hline 20 & & 20 & \multirow{7}{*}{$\begin{array}{l}\text { VERY INTOLERABLE } \\
\text { INTOLERABLE }\end{array}$} \\
\hline 19 & & 19 & \\
\hline 18 & EXTREMELY INTENSE & 18 & \\
\hline 17 & VERY INTENSE & 17 & \\
\hline 16 & \multirow[t]{2}{*}{ INTENSE } & 16 & \\
\hline 15 & & 15 & \\
\hline 14 & STRONG & 14 & \\
\hline 13 & SLIGHTLY INTENSE & 13 & \multirow{3}{*}{$\begin{array}{l}\text { VERY DIS TRESSING } \\
\text { SLIGHTLY INTOLERABLE } \\
\text { VERY ANNOYING } \\
\text { DISTRESSING }\end{array}$} \\
\hline 12 & BARELY STRONG & 12 & \\
\hline 11 & \multirow[t]{3}{*}{ MODERATE } & 11 & \\
\hline 10 & & 10 & VERY UNPLEASANT \\
\hline 9 & & 9 & SLIGHTLY DISTRESSING \\
\hline 8 & \multirow[t]{2}{*}{ MLD } & 8 & \multirow{4}{*}{$\begin{array}{l}\text { ANNOYNG } \\
\text { UNPLEASANT } \\
\text { SLIGHTLY ANNOYNG } \\
\text { SLIGHTLY UNPLEASANT }\end{array}$} \\
\hline 7 & & 7 & \\
\hline 6 & VERY MLD & 6 & \\
\hline 5 & WEAK & 5 & \\
\hline 4 & \multirow[t]{3}{*}{ VERY WEAK } & 4 & \\
\hline 3 & & 3 & \\
\hline 2 & & 2 & \\
\hline 1 & FAINT & 1 & \\
\hline 0 & NO PAIN SENSATTION & 0 & NEUTRAL \\
\hline
\end{tabular}

Figure 2. Gracely Box scale measures of pelvic pain intensity (left) and unpleasantness (right) The Gracely Box Scale (GBS) is a numerical scale that is used to evaluate pain intensity and unpleasantness (GBS pelvic pain intensity and unpleasantness). This scale is comprised of 21 boxes, sequentially numbered beginning with 0 and ending with 20 . It is aligned vertically, with 0 representing the least amount of pain. Descriptive words are arranged next to the numbers corresponding with increasing levels of pain intensity and unpleasantness. 


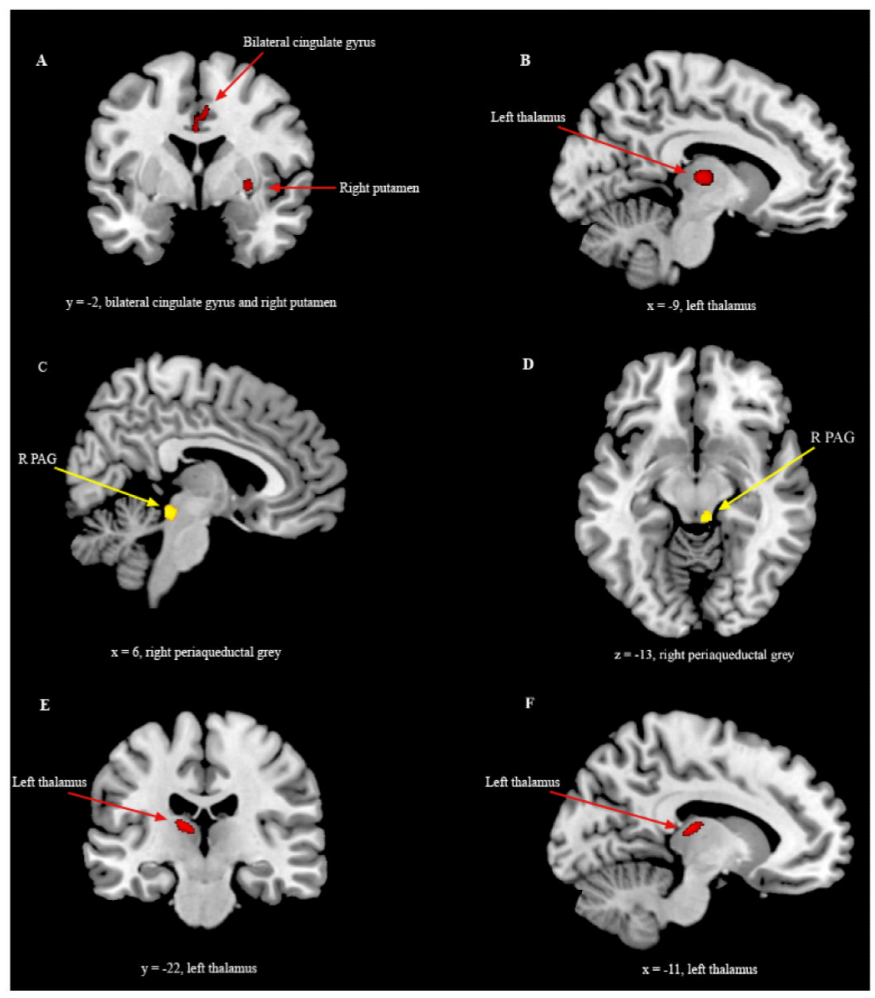

Figure 3. Results from VBM analysis

Statistical parametric maps (SPM) demonstrating differences between groups (t-test with age as a covariate of no interest). Figure A and B: decreased gray matter volumes (in red) in the cingulate gyrus, the right putamen, and the left thalamus in the $\oplus$ Endo $\oplus$ Pain group as compared to healthy controls. Figure $\mathrm{C}$ and $\mathrm{D}$ : increased gray matter volume (in yellow) in the right PAG in the $\oplus$ Endo $\varnothing$ Pain group as compared to healthy controls. Figures Eand F: decreased gray matter volume (in red) in the left thalamus in the $\varnothing$ Endo $\oplus$ Pain group as compared to healthy controls. $\mathrm{P}<.001$, extent threshold $=200$ contiguous voxels. 


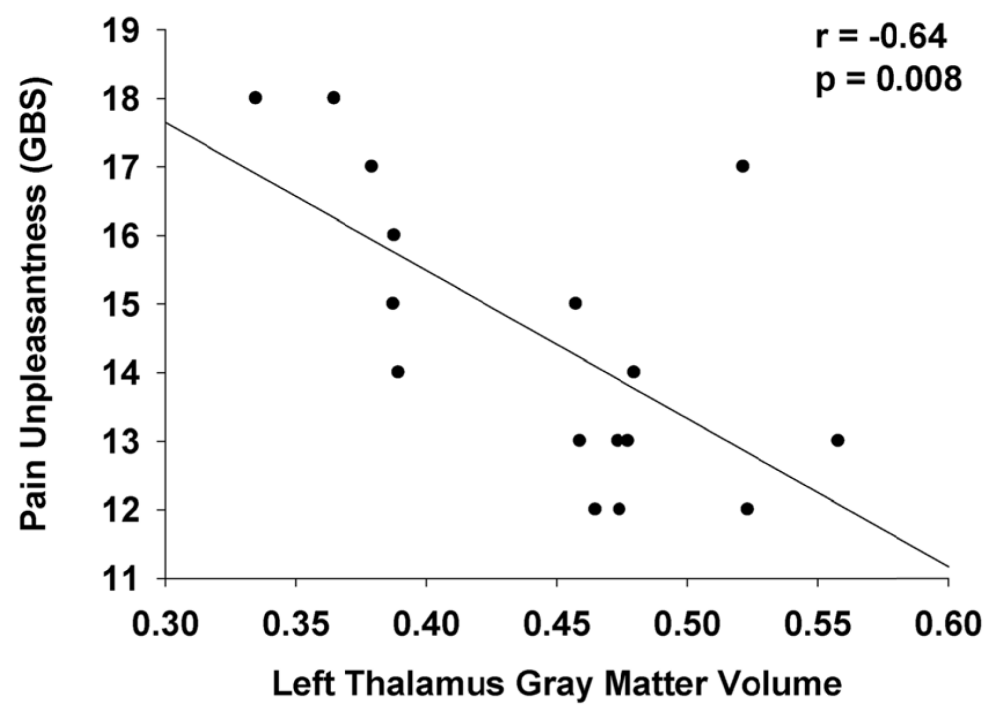

Figure 4. Scatter plot: pain thalamic gray matter vs. pain unpleasantness in the $\bigoplus$ Endo $\oplus$ Pain group

Correlation analysis between left thalamic gray matter (GM), and pelvic pain unpleasantness ratings in the $\oplus$ Endo $\oplus$ Pain group.GM values were extracted from the cluster in the left thalamus identified in the group comparison between the $\oplus$ Endo $\oplus$ Pain group and healthy controls. GBS = Gracely Box Scale, pain unpleasantness in last month. 


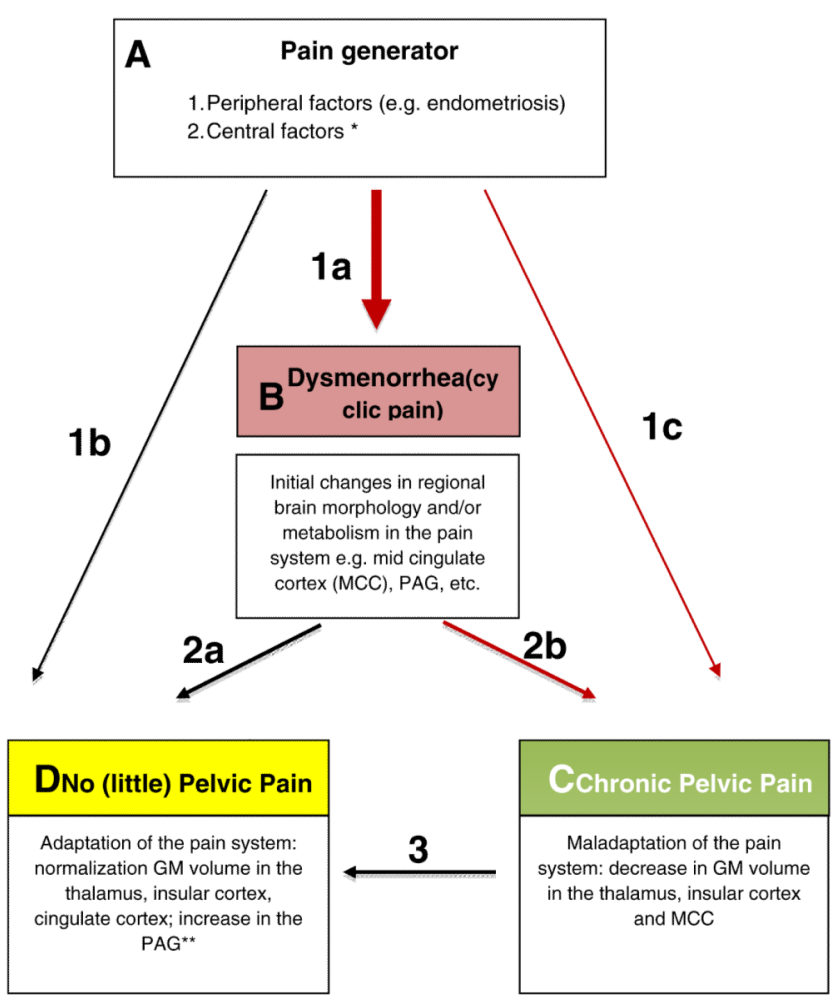

Figure 5. Dysmenorrhea, Endometriosis, and CPP - a conceptual approach to pain chronification

Possible mechanisms of pain chronification in patients with dysmenorrhea and/or endometriosis.Box A:Initially, anociceptive sourceserves as a "pain generator", inducing cyclic pain (dysmenorrhea) and associated changes in regional brain morphology and metabolism.A nociceptive input can be created via sensitized nerve fibers surrounding endometriosis lesions within the pelvis, nerve fiber proliferation in the uterus and/or endometrium (which can also occur in the absence of endometriosis), prostaglandin mediated uterine contractions, or other peripheral abnormalities. ${ }^{*}$ Central factors, such as direct sex hormone-CNS interactions, may also act as pain generators by sensitizing the brain to nociceptive neural input. Box B:Dysmenorrhea and initial changes in regional brain morphology and metabolismmay remain stable or may show further changes, eitheradaptive or maladaptive.Box C: Those women who progress to chronic pelvic pain (CPP) show maladaptive changes in the pain system. Less commonly, this may occur without a preceding phase of dysmenorrhea (arrow 1c). Box D: Those women who experience resolution of their dysmenorrhea (2a) or CPP (3), and those who do not experience pelvic pain despite having a peripheral pain generator (1b) showa "normalization" of or no change in GM volume in the thalamus, insular cortex and cingulate cortex, but have an associated increase in GM volume in the periaqueductal gray (PAG). ** Hypothetically, an increase in GM volume in the PAGis associated with a gain of function of the antinociceptive system. 


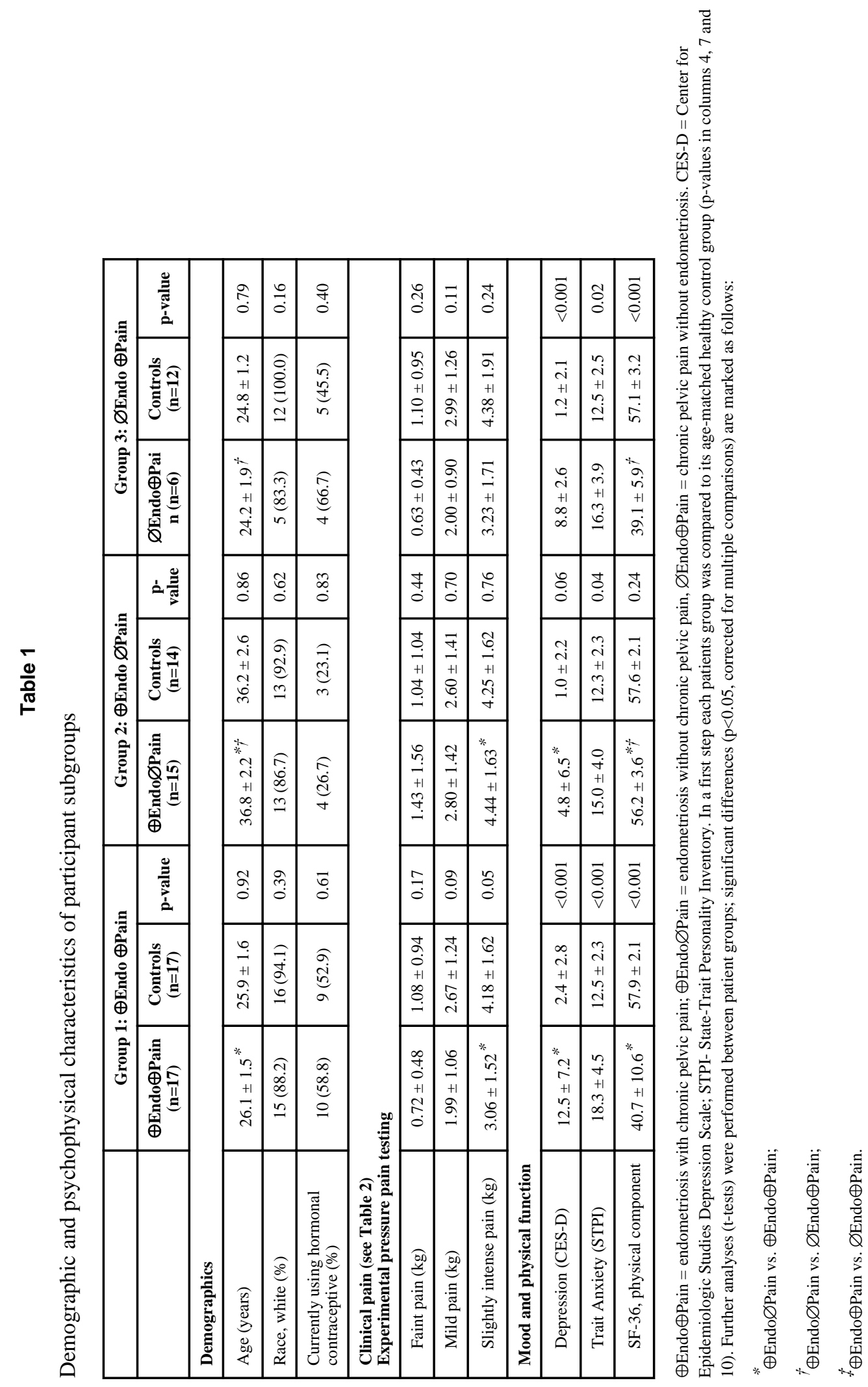

Pain. Author manuscript; available in PMC 2013 May 01. 
Table 2

Clinical characteristics of pelvic pain/endometriosis patients

\begin{tabular}{|c|c|c|c|c|}
\hline & $\begin{array}{c}\text { Group } 1 \\
\oplus \text { Endo } \oplus \text { Pain }\end{array}$ & $\begin{array}{c}\text { Group 2 } \\
\oplus \text { Endo ØРain }\end{array}$ & $\underset{\text { Group } 3}{\varnothing \text { Endo } \oplus \text { Pain }}$ & $\begin{array}{l}\text { ANOVA } \\
\text { 3-group } \\
\text { p-value }\end{array}$ \\
\hline & $(n=17)$ & $(n=15)$ & $(n=6)$ & \\
\hline \multicolumn{5}{|l|}{ Endometriosis stage (rAFS) at most recent surgery } \\
\hline $\mathbf{I}$ & $11(64.7)$ & $4(26.7)$ & 0 & \\
\hline II & $3(7.7)$ & $1(6.7)$ & 0 & \\
\hline III & $1(5.9)$ & $7(46.7)$ & 0 & \\
\hline IV & $2(11.8)$ & $2(13.3)$ & 0 & \\
\hline Pain duration, median years $(95 \% \mathrm{CI})$ & $5.5(3.5,9.5)^{*}$ & $0(0,0)^{* \dagger}$ & $3.75(0.90,9.9)^{\dagger}$ & 0.001 \\
\hline $\begin{array}{l}\text { Months since most recent surgery for endometriosis or pelvic } \\
\text { pain }\end{array}$ & $17.9 \pm 13.5$ & $19.4 \pm 14.7$ & $10.7 \pm 9.8$ & 0.41 \\
\hline \multicolumn{5}{|l|}{ Number prior surgeries for endometriosis or pelvic pain } \\
\hline 1 & $11(64.7)$ & $10(66.7)$ & $5(83.3)$ & \multirow{2}{*}{0.69} \\
\hline$\geq 2$ & $6(35.3)$ & $5(33.3)$ & $1(16.7)$ & \\
\hline Number of pelvic pain days per month, median days $(95 \% \mathrm{CI})$ & $25.0(14.0,29.0)^{*}$ & $0(0,0)^{* \dagger}$ & $25.0(18.4,29.5)^{\dagger}$ & $<0.001$ \\
\hline \multicolumn{5}{|l|}{ Measures of clinical pain intensity } \\
\hline Average pain intensity in last month (GBS, intensity) & $14.8 \pm 2.0^{*}$ & $0.9 \pm 1.9^{* \dagger}$ & $15.3 \pm 2.7^{\dagger}$ & $<0.001$ \\
\hline Average pain unpleasantness inlast month (GBS, unpleasant) & $14.5 \pm 2.1^{*}$ & $0.9 \pm 2.1^{* \dagger}$ & $15.0 \pm 3.0^{\dagger}$ & $<0.001$ \\
\hline Average pain intensity during menses $(0-10)$ & $8.8 \pm 1.4^{*}$ & $1.5 \pm 2.8^{* \dagger}$ & $8.8 \pm 1.3^{\dagger}$ & $<0.001$ \\
\hline
\end{tabular}

$\oplus$ Endo $\oplus$ Pain $=$ endometriosis with chronic pelvic pain; $\oplus$ Endo $\varnothing$ Pain $=$ endometriosis without chronic pelvic pain, $\varnothing$ Endo $\oplus$ Pain $=$ chronic pelvic pain without endometriosis; rAFS = Revised American Fertility Society endometriosis score; GBS = Gracely box scale.

Post hoc analyses were performed to determine significant differences between subgroups, significant differences ( $\mathrm{p}<0.05$, corrected for multiple comparsions) are marked as follows:

* $\oplus$ Endo $\varnothing$ Pain vs. $\oplus$ Endo $\oplus$ Pain;

${ }^{\dagger} \oplus$ Endo $\varnothing$ Pain vs. $\varnothing$ Endo $\oplus$ Pain;

${ }^{*} \oplus$ Endo $\oplus$ Pain vs. $\varnothing$ Endo $\oplus$ Pain 


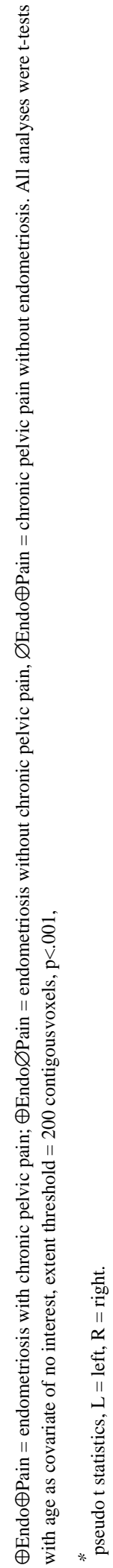

Pain. Author manuscript; available in PMC 2013 May 01. 